\title{
Gastrointestinal amyloidosis complicating psoriatic arthropathy
}

\author{
C. P. WilloughBY* \\ M.A., D.M., M.R.C.P. \\ M. K. BENNETT $\dagger$ \\ M.B. B.S. \\ A. BANERJI* \\ D. P. JEWELL* \\ M.A., B.M. B.Ch. \\ M.A., D.Phil., F.R.C.P. \\ *Gastroenterology Unit and the †Department of Morbid Anatomy, The John Radcliffe Hospital, Oxford, England
}

\begin{abstract}
Summary
A patient is described who developed gastrointestinal amyloidosis complicating psoriatic arthropathy. The presenting symptom was progressive dysphagia due to oesophageal involvement. Other clinical features included gastric ulceration with melaena, intestinal pseudo-obstruction and evidence of impaired renal function. The oesophageal symptoms improved after endoscopic dilatation of the cardia. Colchicine was used in an attempt to slow down progression of the condition.
\end{abstract}

\section{Introduction}

Secondary amyloidosis is associated with a variety of neoplastic and chronic inflammatory disorders (Kyle and Bayrd, 1975), but has rarely been described as a complication of psoriatic arthropathy (Moise, Asch and Imbs, 1965; Ferguson and Downie, 1968; Qureshi et al., 1977). The authors have recently seen a patient with long-standing psoriasis and a seronegative arthritis, who presented with a severe disturbance of gastrointestinal motility and who proved to have extensive amyloidosis.

\section{Case report}

A 35-year-old man was admitted to hospital in 1980 with a 3-month history of heartburn and progressive dysphagia.

He had had severe psoriasis for 17 years, and had been treated with systemic and topical corticosteroids since 1966. Methotrexate, azathioprine, dapsone and hydroxyurea had been tried at different times, all with little benefit. In 1976 he developed a progressive arthritis involving the hands, wrists, elbows, ankles, knees, hips and sacro-iliac joints; a right hip replacement was performed in 1978. In 1977 he was started on methoxsalen $40 \mathrm{mg}$ twice weekly with long-wave ultra-violet light exposure (PUVA therapy); this produced some improvement in his skin condition, and by 1980 he had received almost 200 such treatments. Early in 1979 he was admitted to hospital for intensive therapy of an exacerbation of his skin disease. He had noted alternating diarrhoea and constipation for the previous month. Shortly after admission he developed abdominal pain, vomiting and a fever; plain abdominal X-rays suggested small bowel obstruction. At laparotomy the jejunum and ileum were found to be distended but there was no other abnormality. The abdomen was closed and a prolonged ileus lasting 8 weeks followed.

In 1980 he began to experience difficulty in swallowing; which was worse for solids than for liquids. He had noticed particularly severe retrosternal discomfort after eating fruit or drinking fruit juice, and had avoided these for several months. His dentition was poor and his gums bled easily. $\mathrm{He}$ had become constipated and had lost about $12 \mathrm{~kg}$ in weight.

On examination, he was a thin man with widespread psoriasis and nail pitting. He had extensive joint disease consistent with psoriatic arthropathy, and a right-sided Charnley hip prosthesis. His gums were swollen and friable. Abdominal examination was unremarkable apart from the presence of a poorly healed surgical scar. BP was $140 / 80 \mathrm{mmHg}$ with no postural change and the Valsalva response was normal. His speech was slightly slurred but there was no other abnormality in the central nervous system and no evidence of a peripheral neuropathy.

A few days after admission, the patient began to pass melaena stool and became clinically anaemic.

\section{Laboratory investigations}

Initial $\mathrm{Hb}$ was $13.7 \mathrm{~g} / \mathrm{dl}$, falling to $8.2 \mathrm{~g} / \mathrm{dl}$ after the gastrointestinal haemorrhage. White cell count, platelet count and haematological indices were normal, but the ESR was $68 \mathrm{~mm} / \mathrm{hr}$. Plasma biochemistry was normal apart from a blood urea of 
$9 \cdot 2 \mathrm{mmol} / \mathrm{l}$. Creatinine clearance was $42 \mathrm{ml} / \mathrm{min}$, and $1.14 \mathrm{~g}$ of protein was excreted in the urine over $24 \mathrm{hr}$. No Bence-Jones protein was found, but the urine did contain small amounts of free kappa and lambda light chains. Prothrombin time and liver function tests, including plasma albumin, were normal. Serum $\operatorname{IgA}$ was elevated at $8.3 \mathrm{~g} / \mathrm{l}$ but the other immunoglobulins were normal; serum protein electrophoresis showed a slightly raised $a_{2}$-globulin but no evidence of a paraprotein band. Serum and red cell folate, and serum $B_{12}$ concentrations were all normal. Serum iron was reduced at $8.5 \mu \mathrm{mol} / 1$ and iron-binding capacity was also low at $42 \mu \mathrm{mol} / \mathrm{l}$. A bone-marrow aspirate was normal. Leucocyte ascorbic acid was below detectable levels, and red cell transketolase activity was reduced, suggesting thiamine deficiency. Serological tests for antinuclear factor and rheumatoid factor were negative. The HLA-B27 histocompatibility antigen was not detected.

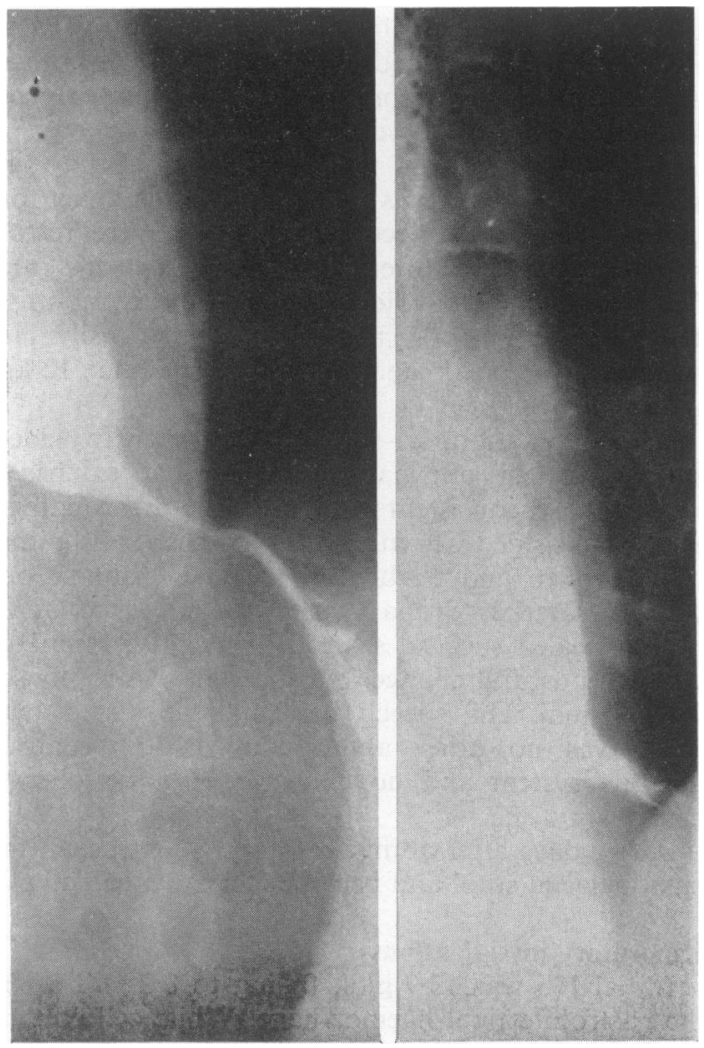

Fig. 1. Barium swallow examination, showing changes consistent with achalasia of the cardia.

\section{Radiology}

A barium swallow had been arranged by the patient's family doctor and was reported to show. the appearances of achalasia of the cardia (Fig. 1 A cinéradiographic examination of swallowing confirmed that the oesophagus was aperistaltic throughout its entire length and that the loweक sphincter failed to relax normally.

The jejunum and ileum were examined by a® intubation small bowel meal. Peristaltic activity was much reduced throughout the small intestine, but the luminal calibre and the appearances of the mucosal folds were normal.

Chest X-ray was normal, but plain abdoming films showed bilateral sacro-iliitis. An i.v. urogra was unremarkable.

\section{Endoscopic findings}

A fibreoptic upper gastrointestinal endoscopy wass performed shortly after the episode of melaena. Th oesophagus was grossly dilated and inflamed, witti large amounts of partly digested food material in tho lumen and patches of thrush on the mucosa. The oesophago-gastric junction was traversed easily? and the stomach was also seen to contain large volumes of undigested food. Peristaltic activity was markedly less than normal and there was an extensive gastritis. The antrum contained 3 small ulcersp one of which was covered by an adherent blood ç] The pylorus and duodenum looked structurā1 normal but, again, peristaltic movements wers sparse.

\section{Histology}

Endoscopic biopsies from the oesophago-gastrit junction showed infiltration of the mucosa wit lymphocytes and polymorphonuclear leucocytes. with superficial ulceration and a few fungal hyphae Within the submucosa were numerous fundal-type glands which were surrounded by acellular eosino 3 . philic material (Fig. 2). After Congo red stainingthis showed the birefringence under cross-polarized. light which is typical of amyloid. The gastric ulceres were histologically benign. The walls of many of the small blood vessels in the gastric and duodena mucosa were completely replaced by amyloid Amyloid was also present as aggregates in the lamina propria and muscularis mucosae of bot organs. Electron microscopy of the duodena deposits confirmed their fibrillary nature (Fig. 3). N

A rectal biopsy was subsequently found to contain small amounts of amyloid material. The papillary vascular plexus of skin from non-psoriatic areas was also affected.

A mesenteric lymph node and a rectal biops $\mathbb{\Phi}$ obtained at the laparotomy 18 months previouslit were reviewed. Both of these had been reported as 


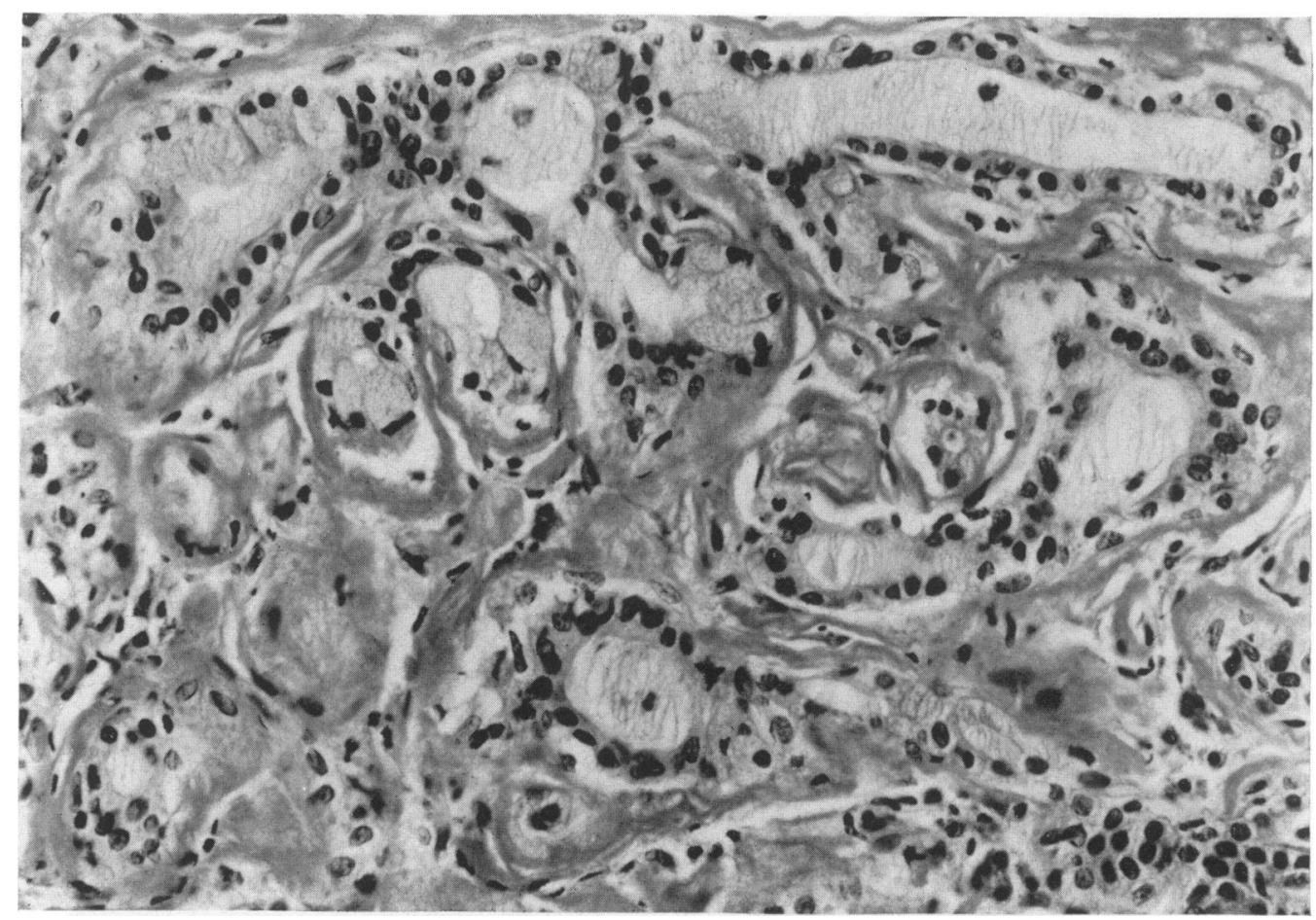

FIG. 2. Biopsy specimen from the oesophagogastric junction. The fundal glands are surrounded by amyloid.

showing no abnormality on routine histological staining, but both in fact contained substantial amyloid deposits.

\section{Treatment and progress}

After the gastrointestinal bleed, the patient was transfused and started on cimetidine. His oesophageal candidiasis was treated with a nystatin suspension. For 2 weeks he was fed enterically using a fine-bore nasogastric tube. When his general nutritional state had improved and his ascorbic acid deficiency had been corrected, the oesophago-gastric junction was gently dilated to $58-\mathrm{F}$ (Charrière) gauge by the endoscopic Eder-Puestow technique (Price, Stanciu and Bennett, 1974), with improvement in his swallowing. He was discharged from hospital taking colchicine $0.5 \mathrm{mg}$ twice daily in an attempt to inhibit further amyloid deposition. He has remained well during the succeeding 6 months, apart from a mild flare-up of his skin condition and a second small gastrointestinal haemorrhage which settled with transfusion and a further course of cimetidine.

\section{Discussion}

Most prior reports suggest that the gastrointestinal tract is involved in well over $50 \%$ of the patients with secondary amyloidosis (Dahlin, 1949; Fentem, Turnberg and Wormsley, 1962; Gilat, Revach and Sohar, 1969). The clinical manifestations of amyloid infiltration include malabsorption (Herskovic, Bartholomew and Green, 1964; Gilat and Spiro, 1968), infarction with perforation (Gilat and Spiro, 1968; Brody, Westlake and Laster, 1964; Griffel, Man and Kraus, 1975), intestinal obstruction (Kyle and Bayrd, 1975) and protein-losing enteropathy (Jarnum, 1965; Hunter et al., 1979). Motility disturbances and bleeding, both of which this patient demonstrated, are particularly common. Oesophageal amyloidosis may result in dysphagia while small intestinal involvement can cause episodic pseudo-obstruction (Gilat and Spiro, 1968; Legge, Wollaeger and Carlson, 1970). Although muscle infiltration has been suggested as the factor responsible for impaired motility, it is possible that neural dysfunction may contribute to this abnormality in some cases (Gilat and Spiro, 1968; French et al., 1965; Battle et al., 1979). This patient showed extensive amyloid deposition in the muscularis mucosae of both stomach and small bowel, but the superficial nature of the tissue biopsies did not allow the authors to assess involvement of the autonomic nerve plexuses. Ulceration of mucosal 


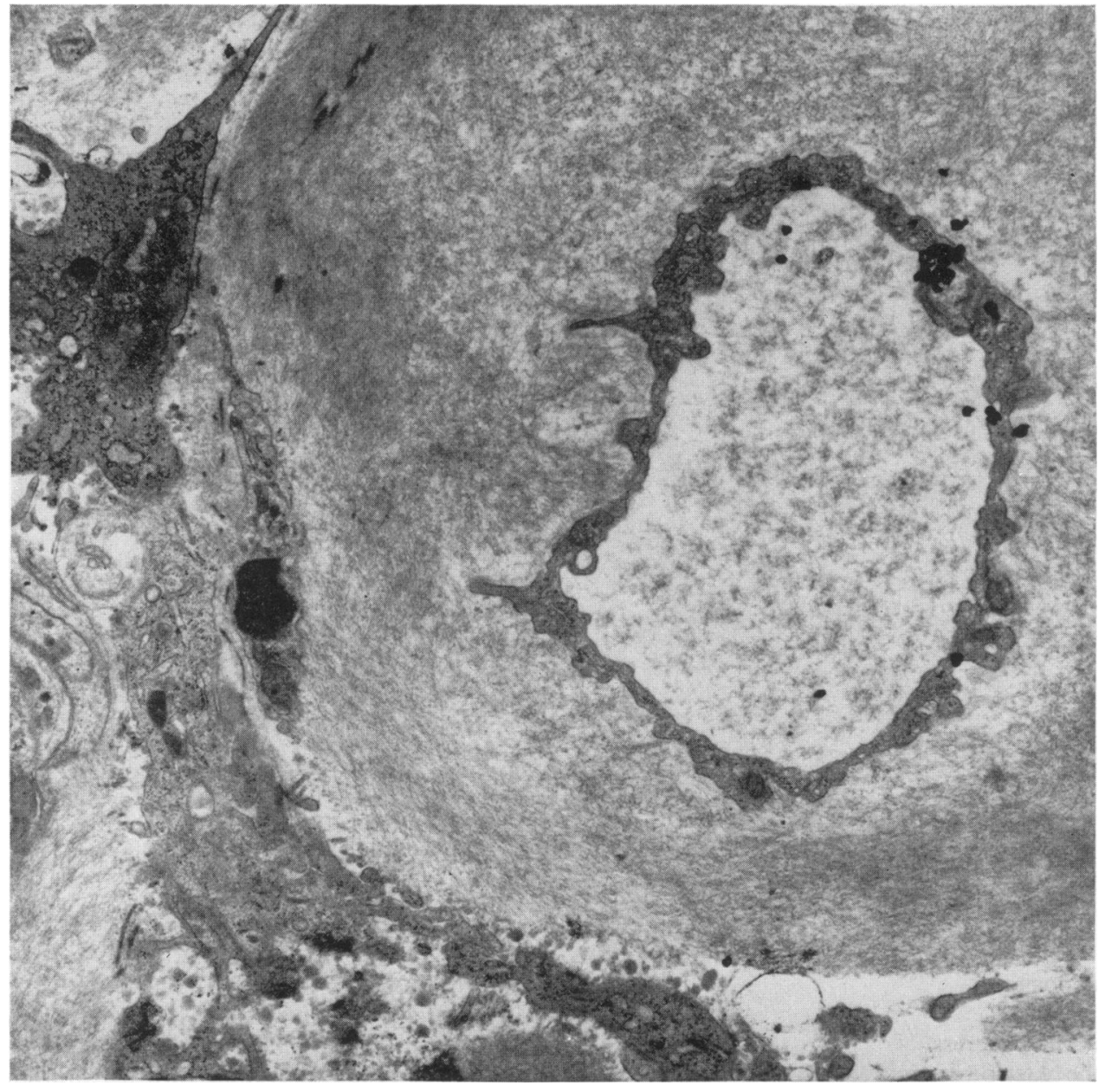

Fig. 3. Electron micrograph of a small blood vessel in the duodenal mucosa. The vessel wall is completely replaced by amylöjd fibrils.

areas containing amyloid material is probably due to local ischaemia secondary to vascular occlusion, and commonly leads to gastrointestinal haemorrhage (Gilat and Spiro, 1968; Brandt, Cathcart and Cohen, 1968). In the present case, the base of the bleeding gastric ulcer contained many blood vessels, the walls of which were almost completely replaced by amyloid.

Skin infiltration has been described in about $40 \%$ of patients with secondary amyloidosis (Rubinow and Cohen, 1978); a biopsy from a non-psoriatic area in the present patient revealed amyloid deposits in the small cutaneous vessels. In a recent paper, Greene and Cox (1979) reported that amyloid was frequently found in the skin of patients with psoriasis who had been treated for prolonged periods with methoxsalen and long-wave u.v. irradiation, ang suggested that it might represent a complication of this form of therapy. However, none of their patients had clinical evidence of systemic involvement, åd the deposits occurred as aggregates in the superficial corium rather than in the perivascular distribution shown by this patient. It therefore seems unlikeby that the PUVA treatment was responsible for the development of extensive amyloidosis in the present case.

Because of the heavy amyloid deposition fougd at the cardia, and also because of the patient's severe ascorbic acid deficiency, the authors were unwill to treat his dysphagia surgically or by vigorots hydrostatic dilatation of the lower oesophagu్s. Dysphagia can be temporarily relieved in patieats 
with achalasia and similar motor disorders by passage of olive dilators through the cardia (Vantrappen and Hellemans, 1980). Eder-Puestow dilatation via a fibreoptic oesophagoscope produced considerable improvement in this patient's swallowing, and this has been maintained over the subsequent months.

In experimental animals, casein-induced synthesis of amyloid can be inhibited by colchicine, possibly because the drug blocks the production of an amyloid-accelerating factor by reticuloendothelial cells (Shirahama and Cohen, 1974; Kedar et al., 1974; Kedar, Greenwald and Ravid, 1976). In theory, colchicine may be beneficial in human amyloidosis by arresting or slowing down progression of the condition, but firm evidence on this point is lacking.

Previous studies suggest that this patient's prognosis is likely to be poor. He has marked proteinuria and an impaired creatinine clearance, which imply the presence of renal amyloidosis. The general experience has been that survival in such cases is usually a matter of a few months from the time of diagnosis (Brandt et al., 1968; Legge et al., 1970; Kyle and Bayrd, 1975).

\section{References}

Battle, W.M. Rubin, M.R., Cohen, S. \& Snape, W.J. (1979) Gastrointestinal motility dysfunction in amyloidosis. New England Journal of Medicine, 301, 24.

Brandt, K., Cathcart, E.S. \& Cohen, A.S. (1968) A clinical analysis of the course and prognosis of forty-two patients with amyloidosis. American Journal of Medicine, 44, 955.

Brody, I.A., Westlake, P.T. \& LASTer, L. (1964) Causes of intestinal symptoms in primary amyloidosis. Archives of Internal Medicine, 113, 512.

DaHLin, D.C. (1949) Secondary amyloidosis. Annals of Internal Medicine, 31, 105.

FenteM, P.H., Turnberg, L.A. \& Wormsley, K.G. (1962) Biopsy of the rectum as an aid to the diagnosis of amyloidosis. British Medical Journal, 1, 364.

Ferguson, A. \& Downie, W.W. (1968) Gastrointestinal amyloidosis in psoriatic arthritis. Annals of the Rheumatic Diseases, 27, 245.
French, J.M., Hall, G., Parish, D.J. \& Smith, W.T. (1965) Peripheral and autonomic nerve involvement in primary amyloidosis associated with uncontrollable diarrhoea and steatorrhoea. American Journal of Medicine, 39, 277.

Gilat, T., ReVach, M. \& Sohar, E. (1969) Deposition of amyloid in the gastrointestinal tract. Gut, 10, 98.

Gilat, T. \& SPIRO, H.M. (1968) Amyloidosis and the gut. American Journal of Digestive Diseases, 13, 619.

Greene, I. \& CoX, A.J. (1979) Amyloid deposition after psoriasis therapy with psoralen and long-wave ultraviolet light. Archives of Dermatology, 115, 1200.

Griffel, B., MaN, B. \& KraUs, L. (1975) Selective massive amyloidosis of small intestine. Archives of Surgery, 110, 215.

Herskovic, T., Bartholomew, L.G. \& Green, P.A. (1964) Amyloidosis and malabsorption syndrome. Archives of Internal Medicine, 114, 629.

Hunter, A.M., Borsey, D.Q., Campbell, I.W. \& Macaulay, R.A.A. (1979) Protein-losing enteropathy due to gastrointestinal amyloidosis. Postgraduate Medical Journal, 55, 822.

JARNUM, S. (1965) Gastrointestinal haemorrhage and protein loss in primary amyloidosis. Gut, 6, 14.

Kedar, I., Greenwald, M. \& Ravid, M. (1976) Colchicine inhibition of the first phase of amyloid synthesis in experimental animals. British Journal of Experimental Pathology, 57, 686.

Kedar, I., Ravid, M., Sohar, E. \& Gafni, J. (1974) Colchicine inhibition of casein-induced amyloidosis in mice. Israel Journal of Medical Sciences, 10, 787.

KYLE, R.A. \& BAYRD, E.D. (1975) Amyloidosis: review of 236 cases. Medicine. Baltimore, 54, 271.

Legge, D.A., Wollaeger, E.A. \& Carlson, H.C. (1970) Intestinal pseudo-obstruction in systemic amyloidosis. Gut, 11, 764.

Moise, R., Asch, L. \& ImBS, J.L. (1965) Rheumatisme psoriasique et amylose. Strasbourg Médecine, 16, 245.

Price, J.D., Stanciu, C. \& Bennett, J.R. (1974) A safer method of dilating oesophageal strictures. Lancet, i, 1141.

Qureshi, M.S.A., Sandle, G.I., Kelly, J.K. \& Fox, H. (1977) Amyloidosis complicating psoriatic arthritis. British Medical Journal, 2, 302.

Rubinow, A. \& Cohen, A. (1978) Skin involvement in generalized amyloidosis. Annals of Internal Medicine, 88, 781.

Shirahama, T. \& Cohen, A.S. (1974) Blockage of amyloid induction by colchicine in an animal model. Journal of Experimental Medicine, 140, 1102.

Vantrappen, G. \& Hellemans, J. (1980) Treatment of achalasia and related motor disorders. Gastroenterology, 79, 144. 